\title{
De quelques incidences de la réforme du droit des contrats sur le bail
}

Laurent IZAC

Maître de conférences en droit privé à l'Université Toulouse 1 Capitole

IEJUC

A proprement parler, la réforme issue de l'ordonnance du 10 février $2016^{1}$ ne concerne pas uniquement notre droit des contrats mais aussi celui du régime général et de la preuve des obligations. Si les changements opérés dans ces deux derniers domaines sont naturellement susceptibles de concerner le contrat de bail -qu'il s'agisse par exemple de le céder à un tiers ou d'établir la preuve de son contenu ${ }^{2}$ - c'est sur les éventuelles incidences de la réforme du droit des contrats sur le bail que nous voudrions centrer notre propos.

Comme on le sait, l'articulation du droit commun et des règles spéciales est régie par l'adage specilia generalibus derogant ${ }^{3}$ voulant que ces dernières l'emportent. Aussi, à considérer rapidement la question des incidences de la réforme du droit des contrats sur le bail, on pourrait penser que ce dernier n'est pas réellement concerné. En effet, fort de ses différents statuts (bail commercial, bail rural, bail d'habitation), il apparait très largement soumis à un droit spécial, un droit que l'on pourrait même qualifier de super spécial.

Cette première impression doit cependant être doublement corrigée.

Tout d'abord, l'organisation du rapport entre règles générales et spéciales implique également que l'empire du droit spécial se cantonne aux seuls sujets dont il s'est emparé et qu'en retour l'autorité des règles générales demeure pour tout ce que de même droit spécial a délaissé.

\footnotetext{
${ }^{1}$ Ordonnance $\mathrm{n}^{\circ}$ 2016-131 du 10 février 2016 portant réforme du droit des contrats, du régime général et de la preuve des obligations.

${ }^{2}$ La preuve du bail connaît cependant un certain nombre de dispositions particulières (art. 1715 et $1716 \mathrm{C}$. civ.).

${ }^{3}$ Art. 1105 C. civ.
} 
Les différents statuts évoqués, aussi nourris soient-ils, n'échappent pas à cette logique. Ainsi, par exemple, s'explique le fait que si, en présence d'un bail commercial, on appliquera les règles relatives à la révision triennale du loyer ${ }^{4}$ prévues par le statut éponyme, en cas de trouble jouissance les règles ordinaires du bail demeureront compétentes tandis qu'en cas de vice du consentement le droit commun des contrats devra être mobilisé. Mais, même dans le cadre de cette articulation du général et du spécial, il conviendra de tenir le plus grand compte du domaine d'application exact des règles concernées. Ainsi, par exemple, si le statut des baux commerciaux organise les conditions de la révision triennale du loyer, l'ordonnance de 2016 a introduit dans notre droit commun des contrats un mécanisme général de révision pour imprévision. L'application de ce dernier, en dehors de l'hypothèse triennale, doit être interrogée. Par ailleurs, même à s'en tenir à l'exemple du statut des baux commerciaux, il faut également garder à l'esprit que toutes ses dispositions ne sont pas d'ordre public. Celles-ci écartées, c'est le droit commun qui constitue le cadre de l'accord des parties.

Ensuite, si les statuts évoqués occupent une place considérable au sein du droit du bail, leurs domaines respectifs, même mis bout-àbout, ne peuvent suffire à couvrir l'ensemble de la matière. Il est en effet des baux qui leur échappent pour relever du droit ordinaire du bail. Tel est le cas du bail mobilier -qui n'intéressera pas notre propos dans le cadre de cette revue- mais aussi des locations « saisonnières » ainsi que des baux « professionnels ${ }^{5}$ » et, dans une moindre mesure, des locations en meublé ${ }^{6}$. Certes, ils relèvent du droit ordinaire du bail, lequel constituent déjà un droit spécial au regard du droit commun des contrats. Mais ce droit ordinaire du bail ne s'intéresse qu'à certaines questions. Si bien qu'il apparaît relativement sporadique. A nouveau, pour tout le reste, c'est le droit commun des contrats qui devra être utilisé.

L'ensemble de ces considérations plaide pour l'intérêt de la question des incidences de la réforme du droit des contrats sur le

\footnotetext{
${ }^{4}$ Art. L. 145-38 C. com.

${ }^{5}$ Seul un article (art. 57 A de la loi du 23 décembre 1986) traite de cette variété de bail et impose essentiellement une durée minimale, une reconduction tacite et un délai de résiliation ouverte uniquement au preneur.

${ }^{6}$ La loi ALUR du 24 mars 2014 a, en effet, rendu applicable un certain nombre de dispositions de la loi du 6 juillet 1989 régissant les baux d'habitation : état des lieux, encadrement des loyers en zone tendue, diagnostic technique, clauses interdites, montant de la commission d'agence, obligations du bailleur et du locataire, colocation, cautionnement, protection des locataires âgés.
} 
bail. A cet égard, il ne saurait être question dans le cadre de cet article de prétendre à l'exhaustivité. Parmi ces incidences, on s'attachera à relever celles qui nous paraissent les plus propices à attirer l'attention du lecteur. Pour ce faire, nous les aborderons lors la formation du bail (I), à propos de son contenu (II), s'agissant de son éventuelle révision (III) et en cas d'inexécution (IV).

\section{LA FORMATION DU BAIL}

Au regard de la formation du contrat de bail, la réforme introduite par l'ordonnance de 2016 est susceptible de produire des incidences de deux ordres : sur les avant-contrats de bail, d'une part, et sur l'information et la bonne foi lors de la conclusion du bail, d'autre part.

En matière d'avant-contrats, le pacte de préférence comme la promesse unilatérale peuvent parfaitement porter sur un bail : soit que priorité ait été contractuellement accordée à un candidat preneur, soit que le droit de conclure unilatéralement celui-ci lui ait été par avance consenti par convention.

S'agissant du pacte de préférence ${ }^{7}$, le régime désormais institué par le Code s'inspire largement des solutions jurisprudentielles jusqu'alors applicables. En cas de violation du pacte au profit d'un tiers, celui-ci ne pourra être inquiété et le bail remis en cause au profit du bénéficiaire du droit de priorité violé que si la double preuve de la connaissance du pacte et de la volonté de son bénéficiaire de s'en prévaloir peut être rapportée. Preuve ô combien exigeante, au point qu'elle a pu être souvent qualifiée de probatio diabolica. Il reste que la réforme innove sur la possibilité offerte au tiers de lever le doute en interrogeant le bénéficiaire sur ses intentions. Bien que particulièrement séduisante, cette procédure interrogatoire n'est qu'une simple faculté à laquelle le tiers peut décider de ne pas recourir. On peut cependant considérer que pareil choix sera malvenu dès lors qu'il aura eu connaissance du pacte. D'une part, son comportement accréditerait l'idée qu'il connaissait ou pressentait la volonté du bénéficiaire de se prévaloir du pacte - facilitant ainsi la tâche du bénéficiaire sollicitant sa substitution dans les liens du contrat. D'autre part, et d'une façon générale, l'abstention du tiers pourrait être considérée comme fautive -parce que déloyale- et source d'un préjudice de perte de

\footnotetext{
${ }^{7}$ Art. 1123 C. civ.
} 
chance au détriment du bénéficiaire : la perte de chance d'user de sa priorité de conclusion du contrat. Incidemment, l'instauration de cette procédure interrogatoire renforce l'efficacité du pacte de préférence.

En matière de bail, on pourrait penser que cette efficacité n'est pas aussi cruciale que lorsqu'est en cause une vente. On objectera que si cette préférence a été sollicitée et obtenue au moyen du pacte, c'est bien la preuve que la conclusion espérée du bail constitue une opportunité importante pour le bénéficiaire. Ainsi, il ne faut pas oublier que la possibilité de louer un local bien situé est d'une très grande importance pour un commerçant, a fortiori, s'il inscrit son projet dans une démarche de développement du maillage territorial de son activité et que le local convoité se situe dans une zone où sont déjà présents ses concurrents.

S'agissant de la promesse unilatérale de contrat ${ }^{8}$, l'innovation est plus évidente encore. Désormais, la violation d'un tel engagement est susceptible d'une sanction en nature. Ecartant la jurisprudence $\mathrm{Cruz}^{9}$, l'ordonnance consacre l'inefficacité de la rétractation du promettant, quand bien même elle aurait été exprimée avant que le bénéficiaire n'ait levé l'option. La possibilité d'obtenir substitution dans le contrat en cas de violation de la promesse au profit d'un tiers est quant à elle conditionnée par la simple connaissance de la promesse par ce tiers. Si cette preuve demeure libre, il reste qu'à la différence de la promesse unilatérale de vente, le recours aux mesures de publicité foncière pour accroitre l'écho de la promesse unilatérale de bail n'est pas évident. Outre la forme notariée qui sera requise pour la convention de promesse unilatérale de bail, il faudra encore qu'elle entre dans l'une des hypothèses visées par le décret de $1955^{10}$. Tel sera certes le cas si la promesse unilatérale porte sur un bail de plus de $12 \mathrm{ans}^{11}$. Mais, en pratique, les baux sont généralement plus courts - comme les baux commerciaux, lesquels sont généralement conclus pour la période légale minimale de 9 ans. Demeurent les baux ruraux de plus longue durée et ayant pu faire l'objet d'une promesse unilatérale. Mais, quoi qu'il en soit, la conclusion d'un bail ne requérant pas en principe la forme notariée, la consultation du fichier immobilier demeurera tout à fait exceptionnelle et, en

\footnotetext{
${ }^{8}$ Art. 1124 C. civ.

${ }^{9}$ Cass. Civ.(3 $\left.3^{\mathrm{e}}\right), 15$ décembre 1993, Bull. civ. III $\mathrm{n}^{\circ} 174$.

${ }^{10}$ Décret $\mathrm{n}^{\circ} 55-22$ du 4 janvier 1955 portant réforme de la publicité foncière.

${ }^{11}$ Art. 37 du décret de 1955 qui en fait une simple faculté destinée à assurer «l'information des usagers » (de la publicité foncière).
} 
conséquence, la mesure de publicité bien peu efficace à l'égard des tiers intéressés par la prise à bail du même bien.

En matière de bonne foi et d'informations délivrées lors de la conclusion du bail, les apports potentiels de la réforme ne doivent pas être sous-estimés.

Certes, dès 1804, le Code civil exigeait la bonne foi dans l'exécution des contrats ${ }^{12}$. De même s'il n'existait pas formellement d'obligation générale d'information, elle était déjà prévue dans le cadre du contrat de vente ${ }^{13}$; contrat majeur s'il en est. Aussi, jouant de son pouvoir d'interprétation, la jurisprudence n'avait pas hésité à en étendre le domaine.

Désormais c'est la nouvelle rédaction du Code civil ${ }^{14}$ qui impose explicitement la présence de la bonne foi aussi bien lors de la négociation que de la formation ou de l'exécution du contrat. De même, est instituée une obligation générale d'information dès lors que son «importance est déterminante pour le consentement de l'autre » partie qui « ignore cette information ou fait confiance à son cocontractant $"{ }^{15}$. Bonne foi et information lors de la formation du contrat ne se confondent pas exactement. Toutefois, c'est à l'aune de la bonne foi que l'on doit mesurer l'étendue de l'information due à l'autre partie. Réciproquement, l'obligation d'information offre un instrument majeur au service du respect de la bonne foi lors de la négociation contractuelle. En matière de bail, l'information du preneur fait déjà l'objet d'une certaine attention du législateur. Ainsi, depuis la loi ALUR de $2014^{16}$, le preneur à bail d'habitation doit-il recevoir toute une série d'informations : indication de la nature et du montant des travaux effectués dans le logement depuis le dernier contrat de location (ou le dernier renouvellement), surface habitable du logement (sous peine de s'exposer à une diminution du loyer), dossier de diagnostic technique. De même, la loi Pinel de $2014^{17}$ a-t-elle considérablement étendu l'information due au preneur à bail commercial. Outre l'établissement obligatoire d'un état des lieux ${ }^{18}$,

\footnotetext{
${ }^{12}$ Anc. art. 1134 al. 3 C. civ.

${ }^{13}$ Aux termes de l'art. 1602 : «Le vendeur est tenu d'expliquer clairement ce à quoi il s'oblige.

Tout pacte obscur ou ambigu s'interprète contre le vendeur. »

${ }^{14}$ Art. 1104 C. civ.

${ }^{15}$ Art. 1112-1 C. civ.

${ }^{16}$ Loi n ${ }^{\circ}$ 2014-366 du 24 mars 2014 pour l'accès au logement et un urbanisme rénové.

${ }^{17}$ Loi $\mathrm{n}^{\circ}$ 2014-626 du 18 juin 2014 relative à l'artisanat, au commerce et aux très petites entreprises.

${ }^{18}$ Dispositif assurant la neutralisation de la présomption de l'art. 1731 C. civ.
} 
le preneur doit être informé de l'état des travaux passés et à venir, du récapitulatif de la répartition des charges, impôts et travaux ${ }^{19}$. $\mathrm{Au}$ travers de ces deux régimes, on aperçoit la volonté du législateur d'inviter les parties à aborder, de la manière la plus transparente possible, les sujets impactant l'équilibre économique $\mathrm{du}$ bail. Les dispositions nouvellement introduites dans le Code civil sont-elles de nature à accroitre un peu plus encore l'obligation d'information des parties en général ${ }^{20}$, et du bailleur en particulier? Il convient d'apporter une réponse en deux temps.

Pour les baux ne relevant pas d'un régime spécial (telles que les locations saisonnières) ou ne faisant l'objet que d'une réglementation embryonnaire (à l'image du bail professionnel), la réponse est sans aucun doute positive. Ainsi, est-il banal de constater que la «qualité » du bien loué est bien souvent surévaluée dans la petite annonce: la mer ou les commerces s'avèrent beaucoup plus loin que prévu, la vue nettement moins dégagée qu'espérée, la présence proche d'une voie express ou d'un chemin de fer passée sous silence, de même la piscine n'est pas réellement privative. Autant d'exemples d'informations que la loi impose désormais au bailleur de fournir au preneur.

Pour les baux spéciaux, on pourrait considérer que l'essentiel a déjà été fait. Mais à mieux se pencher sur la question, il n'est pas impossible de voir dans l'obligation générale d'information du Code civil la source d'exigences complémentaires. Certes, à l'instar de la jurisprudence Baldus ${ }^{21}$, le second alinéa de l'article 1112-1 du Code civil prend le soin d'indiquer que "le devoir d'information ne porte pas sur l'estimation de la valeur de la prestation $»$. Cependant le troisième alinéa de ce texte y intègre, comme étant réputées déterminantes, "les informations qui ont un lien direct et nécessaire avec le contenu du contrat ou la qualité des parties ». Aussi n'est-il pas interdit de voir dans cette disposition la source d'une obligation d'information sur l'état du marché au profit du candidat preneur à bail commercial. Le droit de la franchise nous en donne déjà l'exemple avec l'information précontractuelle issue de la loi Doubin de $1989^{22}$. Certes, il faudra faire le départ entre les bailleurs "particuliers» ne pouvant réellement maîtriser de pareilles informations et les bailleurs

\footnotetext{
${ }^{19}$ Art. L. 145-40-2 C. com.

${ }^{20}$ Pour le preneur, la question se pose aussi. Notamment s'agissant d'éléments de nature à réduire dans le futur sa solvabilité actuelle.

${ }^{21}$ Cass. Civ. $\left(1^{\mathrm{e}}\right), 3$ mai 2000, Bull. civ. I ${ }^{\circ} 131$

${ }^{22}$ Loi $\mathrm{n}^{\circ}$ 89-1008 du 31 décembre 1989 relative au développement des entreprises commerciales et artisanales et à l'amélioration de leur environnement économique, juridique et social.
} 
«professionnels » - telles que les grandes surfaces louant des locaux dans leurs galeries commerciales. Ces derniers, à l'image des franchiseurs, disposent d'une lecture fine du marché. Ne seraitce que grâce à l'étude préalable à leur propre implantation.

\section{LE CONTENU DU BAIL}

Le contenu du contrat en général et de celui de bail en particulier est susceptible d'être doublement encadré par les dispositions nouvelles figurant aux articles 1170 et 1171 du Code civil.

Le premier de ces deux textes apparaît nettement comme la consécration légale de la jurisprudence Chronopost ${ }^{23}$ relative aux clauses limitatives de responsabilité. L'article 1170 dispose en effet que "toute clause qui prive de sa substance l'obligation essentielle du débiteur est réputée non écrite ». Officiellement disparue en tant que condition générale de validité du contrat, la cause reparaît ici par le biais de l'exigence de cohérence contractuelle : il apparaît logique qu'on ne puisse s'engager tout en prévoyant par ailleurs que sa défaillance, aussi grave soit-elle, ne pourra être source de responsabilité à l'égard de son partenaire contractuel. En matière de bail, cette disposition prend une coloration particulière pour deux raisons.

Tout d'abord, il convient de relever que les articles 1719 et 1720 du Code civil prévoient que « le bailleur est obligé, par la nature $d u$ contrat, et sans qu'il soit besoin d'aucune stipulation particulière :

$1^{\circ}$ De délivrer au preneur la chose louée et, s'il s'agit de son habitation principale, un logement décent. Lorsque des locaux loués à usage d'habitation sont impropres à cet usage, le bailleur ne peut se prévaloir de la nullité du bail ou de sa résiliation pour demander l'expulsion de l'occupant;

$2^{\circ} D^{\prime} e n t r e t e n i r$ cette chose en état de servir à l'usage pour lequel elle a été louée :

$3^{\circ} D^{\prime} e n$ faire jouir paisiblement le preneur pendant la durée $d u$ bail ;

$4^{\circ} D^{\prime}$ assurer également la permanence et la qualité des plantations " et que "le bailleur est tenu de délivrer la chose en bon état de réparations de toute espèce. Il doit y faire, pendant la durée $d u$ bail, toutes les réparations qui peuvent devenir nécessaires, autres que les locatives. "

${ }^{23}$ Cass. Com., 22 octobre 1996, Bull. Civ. IV nº 261. 
Or, si par le passé la jurisprudence a pu considérer que certaines de ces dispositions pouvaient être écartées au motif qu'elles n'étaient pas d'ordre public ${ }^{24}$, on peut aujourd'hui douter de la pérennité de cette solution. En effet, si le domaine de la jurisprudence Chronopost se limitait aux clauses limitatives ou élusives de responsabilité, les dispositions de l'article 1171 du Code civil ne s'y cantonnent pas. Dès lors, si l'on considère que les obligations visées par les articles 1719 et 1720 du Code civil participent de l'essence du bail, elles ne devraient plus pouvoir être écartées. A moins cependant d'établir que l'équilibre financier du bail a été expressément calculé pour tenir compte de cet aménagement contractuel. On pense, par exemple, à un bien que le preneur se serait chargé de remettre en état et même d'entretenir par la suite au-delà des seules « réparations locatives », moyennant un loyer réduit en conséquence. Il est vrai, pour en revenir à la cause laquelle sous-tend le raisonnement- que la jurisprudence Chronopost devait elle-même être nuancée par la solution issue des arrêts Faurecia ${ }^{25}$; laquelle était destinée à permettre aux juges du fond de se prononcer par une appréciation in concreto de la portée de la clause limitative de responsabilité sur l'économie générale du contrat.

Ensuite, c'est justement cette éventuelle modification de la répartition de la charge des réparations entre bailleur et preneur qui donne lieu à une réglementation spéciale et impérative. Présente de longue date en matière de baux d'habitation ${ }^{26}$, elle est apparue dans les baux commerciaux à l'occasion du décret d'application de la loi Pinel de $2014^{27}$. Désormais, la pratique jusqu'alors courante qui consistait à reporter contractuellement la charge des grosses réparations sur le preneur à bail commercial est prohibée.

Est-ce à dire qu'il faudrait voir dans ces dispositions spéciales l'application sectorielle d'un principe général que l'article 1171 imposerait désormais à tous les baux ${ }^{28}$ ? Sous réserve de

\footnotetext{
${ }^{24}$ Ainsi, par exemple, de la validité de la clause écartant la responsabilité du bailleur pour les nuisances dues à des travaux sur les parties communes d'un immeuble dans lequel étaient loués des bureaux : Cass. Civ.( $\left.3^{\mathrm{e}}\right), 30$ mai 1996, CCC 1996, p. 185.

${ }^{25}$ Cass. Com., 13 février 2007, Bull. Civ.IV n ${ }^{43}$. Cass. Com., 29 juin 2011 , Bull. Civ. IV n ${ }^{\circ} 115$.

${ }^{26}$ Décret $n^{\circ} 87-712$ du 26 août 1987 pris en application de l'article 7 de la loi ${ }^{\circ}$ 86-1290 du 23 décembre 1986 tendant à favoriser l'investissement locatif, l'accession à la propriété de logements sociaux et le développement de l'offre foncière et relatif aux réparations locatives.

${ }^{27}$ Art. R. 145-35 C. com.

${ }^{28}$ Rapp. H. Kenfack, Liberté contractuelle et clauses de transfert de charges, AJDI 2014, n², p. $101 \mathrm{~s}$.
} 
l'appréciation in concreto évoquée, il est permis de penser qu'interdire les dérogations contractuelles aux dispositions des articles 1719 et 1720 du Code civil participerait d'une amélioration de la lutte contre les bailleurs indélicats ${ }^{29}$.

Second mécanisme à évoquer, celui instauré par l'article 1171 vient opportunément compléter le dispositif du texte précédent. En effet, la notion de cause à l'œuvre dans l'article 1170 ne permet pas de remédier aux déséquilibres contractuels issus de clauses relatives à des obligations non essentielles. Or, bien que plus périphériques ou accessoires, certaines clauses sont susceptibles de permettre à l'une des parties de tirer un profit excessif du contrat au détriment de l'autre. Ce sont justement contre ces clauses que le dispositif introduit dans l'article 1171 du Code civil se propose de lutter au moyen d'un mécanisme de censure des clauses abusives largement inspiré de celui déjà présent en droit de la consommation.

Si dans le projet initial un article 1169 tendait à généraliser le dispositif de l'article L. 212-1 du Code de la consommation, les critiques exprimées par une partie de la doctrine et des milieux professionnels ont conduit le rédacteur de l'ordonnance à en réduire le domaine d'application aux seuls contrats d'adhésion. La lutte contre les clauses abusives n'est ainsi ouverte sur le fondement de ce texte que si la clause contestée provient d'un tel contrat d'adhésion.

L'applicabilité de l'article 1171 en matière de bail exige de la sorte le respect de cette condition préalable. Or, aux termes de l'article 1110 , le contrat d'adhésion « est celui dont les conditions générales, soustraites à la négociation, sont déterminées à l'avance par l'une des parties ». S'il ne fait aucun doute que le recours à ce procédé ait été quasi-systématique pour certains baux tels que les baux d'habitation, au point que le législateur ait décidé d'imposer un contrat type règlementaire ${ }^{30}$-ce qui règle la question pour cette variété de baux ${ }^{31}$-, il reste que nombre de situations relèvent d'une «zone intermédiaire entre le vrai contrat de gré à gré et le pur contrat d'adhésion $»^{32}$. C'est le cas relativement courant des baux

\footnotetext{
${ }^{29}$ Pareille solution apparaît en partie préfigurée dans la jurisprudence invalidant la clause permettant, de fait, au bailleur de priver son preneur de toute jouissance sans qu'il puisse prétendre en être indemnisé : Cass. Civ. $\left(3^{\mathrm{e}}\right), 1^{\mathrm{er}}$ juin 2005 , Bull. civ. III $n^{\circ} 119$.

${ }^{30}$ Décret $n^{\circ} 2015-587$ du 29 mai 2015 relatif aux contrats types de location de logement à usage de résidence principale.

${ }^{31}$ L'usage de ce contrat type est imposé depuis le $1^{\text {er }}$ aout 2015.

${ }^{32}$ S. Gaudemet, Quand la clause abusive fait son entrée dans le Code civil, C.C.C. 2016 , dossier 5 .
} 
commerciaux, en partie négociés et en partie imposés en fonction du contexte économique et des moyens des parties. En présence de ces situations hybrides, l'application de l'article 1171 nous semble pertinente dès lors que l'article 1110 n'exige pas que l'intégralité du contrat d'adhésion soit faite de conditions générales nonnégociées, tandis qu'une logique d'application distributive commande justement que ces clauses non-négociées puissent tomber sous le coup de la censure prévue par l'article $1171 \mathrm{du}$ Code civil. Si l'on admet ce raisonnement, il reste encore à définir les critères du caractère abusif de la clause. Dans ses arrêts récents, la Cour de cassation pose le principe d'un contrôle de la nécessité et de la proportionnalité de la clause contestée. A cette occasion, elle édicte même une présomption en défaveur du stipulant puisqu'elle lui impose de démontrer que la clause litigieuse « est nécessaire à l'équilibre de la convention ou que ce déséquilibre est compensé par d'autres dispositions du contrat $\gg{ }^{33}$. Qu'en est-il en matière de baux commerciaux ? Comme le relève un auteur ${ }^{34}$, les exemples sont relativement nombreux : clause par laquelle le bailleur, qui a certes la possibilité de vendre l'immeuble, s'autorise également à procéder, en cours de bail, à une opération de leaseback, laquelle fait du preneur un sous-locataire sans son accord; clause qui exclut toute garantie de commercialité et écarte ainsi toute responsabilité du bailleur en cas de désertification du centre commercial alors qu'il en est le seul maître ; clause permettant au bailleur d'effectuer toute catégorie de travaux, de modifier le bien, ses accessoires, son environnement, voire de le transférer, le tout sans indemnité, quelle que soit la durée des travaux. En dehors de ces illustrations tirées des baux commerciaux, on peut également citer la clause, très courante en matière de location saisonnière, permettant au bailleur d'exiger ou de conserver le paiement de l'intégralité du séjour tout en se réservant la possibilité de louer le bien à un tiers en cas d' « annulation » tardive.

Enfin, une dernière difficulté doit être évoquée en matière de baux commerciaux. Une disposition particulière visant les clauses abusives est déjà prévue par le Code de commerce ${ }^{35}$, depuis qu'elle y a été introduite par la loi LME de $2008^{36}$. Toutefois, celle-ci ne concerne pas les mêmes hypothèses (contrat d'adhésion dans le Code civil, rapport de soumission dans le Code de commerce), ni

\footnotetext{
${ }^{33}$ Cass. com., 29 septembre 2015, $\mathrm{n}^{\circ}$ 13-25.043: JCP E 2016, 1040, note S. Legac-Pech.

${ }^{34}$ Voir l'étude très fouillée de F. Auque, Bail commercial et contrôle $d u$ déséquilibre significatif, Loyers et Copropriété $\mathrm{n}^{\circ}$ 10, Octobre 2016, dossier 11.

${ }^{35}$ Art. L. $442-6, \mathrm{I}, 2^{\circ} \mathrm{C}$. com.

${ }^{36}$ Loi n ${ }^{\circ} 2008-776$ du 4 août 2008 de modernisation de l'économie.
} 
n'organise la même sanction (nullité de la clause abusive dans le Code civil, paiement de dommages et intérêts dans le Code de commerce). L'application alternative, voire combinée, des dispositions du Code civil et du Code de commerce demeure ainsi possible $^{37}$.

\section{LA RÉVISION DU BAIL}

Longtemps écartée en vertu la jurisprudence du Canal de Craponne $^{38}$, l'imprévision a été introduite par l'ordonnance à l'article 1195 du Cde civil. Elle permet, sous un certain nombre de conditions, d'infléchir le principe de l'intangibilité contractuelle afin de permettre à la partie qui vient à trop souffrir d'un contrat d'en obtenir la révision.

Le mécanisme nouvellement institué prend la forme d'une demande de renégociation contractuelle que l'article 1195 admet à la triple condition: 1) d'un changement de circonstances imprévisibles lors de la conclusion du contrat, 2) que ces circonstances rendent l'exécution du contrat excessivement onéreuse pour l'une des parties, 3) que cette dernière n'ait pas par avance accepté d'en assumer le risque. Ce même texte prévoit qu'à défaut d'accord dans la renégociation du contrat, le juge pourra réviser le contrat.

Même s'il n'est pas absolument inconcevable qu'il s'applique à un bail professionnel ou même à un bail d'habitation, c'est principalement les baux organisant l'exploitation de l'immeuble par le preneur que ce texte intéresse. Pour le preneur à bail rural ou commercial, un changement imprévu de circonstances peut mettre à mal l'équilibre financier de son activité.

Avant de s'intéresser à de telles circonstances, il convient toutefois d'évoquer la possibilité qu'auraient les parties d'écarter le jeu de l'article 1195. En effet, comme de nombreux auteurs n'ont pas manqué de le relever, le rapport au Président de la République ${ }^{39}$ indique expressément que les dispositions de ce texte sont supplétives. La pratique n'a pas manqué de s'appuyer sur cette idée puisque, depuis l'entrée en vigueur de l'ordonnance, les clauses écartant les dispositions de l'article 1195 ont fait flores dans les contrats. L'efficacité de cette parade appelle toutefois quelques

\footnotetext{
${ }^{37}$ En ce sens, F. Auque, préc., M. Mekki, L'ordonnance $n^{\circ} 2016-131 d u 10$ février 2016 portant réforme du droit des contrats, du régime général et de la preuve des obligations, D. 2016, p. 494.

${ }^{38}$ Cass. civ., 6 mars 1876, DP 76. 1. 193.

${ }^{39} \mathrm{JORF}^{\circ} 0035$ du 11 février 2016, texte $\mathrm{n}^{\circ} 25$.
} 
précisions. En effet, la possibilité d'écarter le jeu de la révision pour imprévision réside dans l'éventuelle acceptation du risque contractuel: outre la lésion, l'aléa (accepté) chasse aussi l'imprévision. C'est en ce sens spécifique que l'article 1195 présente un caractère supplétif. Il nous semble donc excessif d'affirmer que l'article 1195 serait supplétif au sens qu'il pourrait être écarté " en bloc » grâce à l'accord indifférencié des parties. $\mathrm{Au}$ contraire, le texte réserve son éviction à la survenance imprévue des seules circonstances pour lesquelles le risque a été par avance accepté. En d'autres termes, comme le rapport prend le soin de l'évoquer "les parties pourront convenir à l'avance [d'écarter ce mécanisme] pour choisir de supporter les conséquences de la survenance de telles circonstances qui viendraient bouleverser l'économie du contrat $\gg{ }^{40}$. A une éviction globale et abstraite de l'imprévision, l'article 1195 préfère une éviction ponctuelle et concrète, circonscrite dans l'accord circonstancié des parties.

S'agissant des baux, la précision est d'importance parce que le déséquilibre contractuel peut résulter de circonstances très différentes. Il peut s'agir tout d'abord de circonstances économiques: aussi bien macro-économiques (comme une dévaluation monétaire) que micro-économiques (modification structurelle du marché local). Il peut s'agir ensuite de circonstances matérielles (catastrophe climatique, conflit armé, acte terroriste). Il peut s'agir enfin de circonstances juridiques (restrictions ou prohibitions nouvelles). Il appartiendra aux parties de clairement convenir des circonstances dont l'une et/ou l'autre -on pense essentiellement au preneur- accepteront par avance de courir le risque.

En matière de baux commerciaux ${ }^{41}$, la présence d'un mécanisme légal de révision doit également être évoquée. L'article L. 145-38 du Code de commerce ouvre la faculté de solliciter une révision triennale s'appuyant sur la variation d'ILC durant la période considérée. Toutefois, si le loyer a été affecté d'une clause d'échelle mobile, l'article L. 145-39 du Code de commerce n'ouvre de faculté de révision que si le jeu de ladite clause a conduit à une augmentation de plus d'un quart par rapport au prix précédemment fixé contractuellement ou par décision judiciaire.

Qu'en est-il de l'articulation de ces régimes spéciaux de révision avec le mécanisme général institué par l'article 1195 au titre de l'imprévision? La question se pose d'autant plus que tous ces

\footnotetext{
${ }^{40}$ C'est nous qui soulignons.

${ }^{41}$ Le loyer du bail rural (fermage) fait l'objet d'une réglementation légale organisant son actualisation annuelle : art. L. 411-11 C. rur.
} 
mécanismes partagent le même objet: assurer l'adaptation du loyer aux circonstances intervenues depuis la conclusion du bail. En raison de cette communauté d'objet, doit-on considérer que les règles de révision $\mathrm{du}$ Code de commerce évincent automatiquement celles prévues par le Code civil au titre de l'imprévision?

La réponse à cette question dépend de la façon dont le Code de commerce organise la révision. Le mécanisme de révision triennale de l'article L. 145-38, en s'appuyant sur un critère temporel, assure une adaptation «ordinaire » du loyer : tous les trois ans le loyer peut être ajusté pour tenir compte de l'évolution générale des loyers commerciaux ${ }^{42}$. Aux côtés de cette adaptation « ordinaire » programmée dans le temps par l'article L. 145-38 du Code de commerce, l'article 1195 du Code civil devrait permettre d'ajouter une adaptation «exceptionnelle » à la survenance imprévisible d'un événement extraordinaire. Révision triennale et révision pour imprévision pourraient ainsi se compléter en fonction des circonstances.

Le mécanisme de révision ouvert par l'article L. 145-39 s'appuie quant à lui sur une variation quantitative du loyer dont l'importance justifie l'éventuelle adaptation d'un montant jusqu'alors mécaniquement ajusté. On doit logiquement en déduire qu'en présence d'une clause d'échelle mobile l'ajustement automatique du loyer est réputé satisfaisant dès lors qu'il ne dépasse pas un certain seuil de variation. En d'autres termes, les parties sont réputées avoir anticipé l'avenir au moyen d'une indexation pertinente, et cela dans la limite de la variation excessive évoquée. Le recours au mécanisme de l'article 1195 du Code civil devient dès lors plus problématique. Il nous semble cependant demeurer applicable parce que, sous certaines conditions $^{43}$, le Code de commerce admet le recours à la révision triennale nonobstant la présence d'une clause d'échelle mobile. Or, réserver cette possibilité, c'est sous-entendre que le jeu de la clause d'échelle mobile peut parfois ne pas être suffisamment adapté à l'évolution des circonstances. Le recours à la révision pour imprévision pourrait demeurer envisageable en ce cas, même si c'est au prix d'une plus grande exigence du juge quant à la preuve de l'absence d'acceptation du risque contractuel.

\footnotetext{
${ }^{42}$ Sauf circonstances locales (telles que définies par ce même texte) justifiant par leur ampleur une révision plus conséquente du loyer (déplafonnement).

${ }^{43}$ Le mécanisme légal de la révision triennale demeure applicable si, d'une part, une modification matérielle des facteurs locaux de commercialité a entrainé une variation de plus de $10 \%$ de la valeur locative, et d'autre part, un délai de 3 ans s'est écoulé depuis la dernière fixation amiable ou judiciaire du loyer.
} 


\section{L’INEXÉCUTION DU BAIL}

Le nouveau régime de l'inexécution contractuelle recèle plusieurs dispositions susceptibles d'intéresser le droit du bail. Elles sont de quatre ordres.

En premier lieu, il convient d'évoquer la résolution à l'initiative du créancier s'estimant victime d'une inexécution suffisamment grave pour justifier qu'il rompe le contrat. Ce mécanisme institué par l'article 1226 vient s'insérer entre les dispositifs classiques de la clause résolutoire et de la résolution judiciaire. Il s'appuie sur la seule appréciation du créancier dont l'initiative de rompre le contrat se fait alors à «ses risques et périls». Consacrant le mécanisme que la Cour de cassation avait déjà déduit de l'ancien article $1184^{44}$, l'ordonnance y apporte plusieurs compléments : l'exigence de mise en demeure préalable du débiteur -sauf urgence- et celle de notifier les motifs de la résolution décidée par le créancier insatisfait.

A l'égard des baux pour lesquels la loi règlemente strictement le jeu des clauses résolutoires, les dispositions de l'article 1226 ouvrent de prime abord d'importantes perspectives au bailleur désireux de libérer son bien d'un preneur défaillant. En réalité, la portée pratique de ce texte doit être fortement nuancée pour deux raisons. Tout d'abord, cette rupture unilatérale demeure soumise à l'appréciation du juge. En effet, sous la seule différence qu'il rendra un jugement déclaratif (validant ou invalidant la rupture du bail), le juge conservera pleinement son pouvoir d'appréciation en opportunité pour décider si le manquement reproché était bel et bien suffisamment grave pour justifier la rupture prétendue du contrat. En d'autres termes, le régime de cette rupture demeurera extrêmement proche de celui résultant d'une résiliation judiciaire « ordinaire » : à une résiliation judiciairement prononcée, l'article 1226 permet de substituer une résiliation judiciairement validée (ou invalidée). Ensuite, les baux règlementés connaissent des dispositions propres à la résiliation et à la clause résolutoire. Ainsi, pour le bail d'habitation, l'article $4 \mathrm{~g}$ de la loi du 6 juillet 1989 restreint très fortement le domaine de la clause résolutoire à une liste limitative d'hypothèses objectives ${ }^{45}$. En creux, ce texte

\footnotetext{
${ }^{44}$ Cass. Civ.(1 $\left.{ }^{\mathrm{e}}\right), 13$ octobre 1998, Bull. Civ. I n 300.

45 Seules cinq circonstances sont admises (la dernière étant d'ailleurs une hypothèse judiciarisée) : non-paiement du loyer, des charges, du dépôt de garantie, la non-souscription d'une assurance des risques locatifs ou le non-
} 
laisse entendre qu'aux yeux du législateur les manquements visés sont a priori les seuls suffisamment graves pour justifier une rupture déjudiciarisée du bail. Certes, il ne s'agit que d'une indication générale qui n'interdit pas d'envisager des hypothèses autres, subjectives et graves, telle celle d'un preneur ayant causé d'importantes dégradations au bien loué. Il reste que l'intention initiale du législateur exercera une certaine influence sur l'appréciation du juge. Par ailleurs et encore, ces mêmes dispositions spéciales restreignent l'efficacité du jeu de la clause résolutoire ${ }^{46}$ en ne lui faisant produire effet qu'après un certain délai : deux mois pour le bail d'habitation ${ }^{47}$ et un mois pour le bail commercial $^{48}$. Par analogie, on doit en déduire que la mise en demeure adressée au débiteur défaillant devra a minima reprendre ce délai avant que la résiliation par notification de l'article 1226 ne puisse prétendre produire effet. En définitive, et contre toute attente, c'est peut-être du côté du preneur que ce texte offrira le plus de perspectives. Si tant est qu'il souhaite se défaire du bail en réaction aux graves manquements du bailleur (refus de procéder à des réparations importantes, violation de la clause d'exclusivité commerciale). En règle générale, il préfèrera opter pour l'exécution forcée ou la réduction du loyer.

En second lieu, la réforme organise le recours à l'exécution forcée en nature. Outre l'exécution forcée en nature proprement dite, désormais consacrée par l'article 1221 -prenant ainsi le contrepied direct des dispositions de l'ancien article 1142-, l'article 1222 confirme la faculté de remplacement déjà présente dans l'ancien article 1144. Ainsi, en matière de baux, le recours au mécanisme des travaux «à frais avancés » demeure d'actualité. On sait qu'il peut s'avérer particulièrement utile pour le preneur se heurtant à l'inertie d'un bailleur peu enclin à réaliser les travaux qui pourtant lui incombent.

Mais l'article 1144 innove en admettant que le créancier puisse, sans obtenir l'autorisation préalable du juge, prendre l'initiative de réaliser ou de faire réaliser l'obligation du débiteur défaillant. Le

respect de l'obligation d'user paisiblement des locaux loués, résultant de troubles de voisinage constatés par une décision de justice passée en force de chose jugée.

${ }^{46}$ En matière de baux ruraux, la loi n'admet que la résiliation judiciaire (art. L. 411-30 s. C. rur.). Il faut toutefois relever que 1'art. L. 411-32 C. rur. organise une sorte de clause résolutoire légale visant le changement d'affectation des parcelles louées (passage en zone urbaine dans la réglementation locale de l'urbanisme). La résiliation ne produit cependant effet que passé un an après que le bailleur s'en soit prévalu à l'égard du preneur.

${ }^{47}$ Art. 24 loi de la loi du 6 juillet 1989, préc.

${ }^{48}$ Art. L. 145-41 C. com. 
recours au mécanisme évoqué se trouve ainsi renforcé, moyennant cependant une prise de risque pour le preneur ayant fait le choix de ne pas solliciter d'autorisation judiciaire. En contrepartie, il offre une alternative intéressante face aux restrictions que la jurisprudence $^{49}$ pose au recours à l'exception d'inexécution en matière de baux.

En troisième lieu, l'ordonnance introduit à l'article 1223 une action en réduction de prix. La réfaction du contrat fait ainsi son entrée officielle dans le droit commun du contrat alors qu'elle était à l'origine plutôt l'apanage du droit commercial. En matière de baux, le prix réside dans le loyer. Le recours éventuel aux dispositions de l'article 1223 n'appartiendra donc qu'au preneur souhaitant obtenir une minoration de loyer en raison des manquements du bailleur. L'hypothèse déjà évoquée d'un défaut de réparation en offrira sans aucun doute le meilleur exemple. Pour ce qui est de son domaine d'application, l'action en réduction du loyer se trouve désormais ouverte à tout preneur confronté à un manquement du bailleur. A ce titre, elle ne se substitue, ni ne contredit les autres actions spéciales en réduction de loyer que la loi connaît déjà ${ }^{50}$.

En quatrième et dernier lieu, il convient d'aborder la définition nouvelle de la force majeure en présence d'un contrat. Désormais, la notion se décline différemment en fonction du contexte contractuel ou extracontractuel ${ }^{51}$. La subjectivisation qui en résulte permet de tenir compte de l'accord des parties dans l'anticipation des évènements pouvant par la suite rendre impossible l'exécution du contrat. On retrouve ainsi la question de l'acceptation des risques contractuels évoquée à propos de l'imprévision.

\footnotetext{
${ }^{49}$ Elle n'est admise que si la jouissance du preneur est totalement impossible ou très gravement compromise : Cass. Civ.( $\left.3^{\mathrm{e}}\right), 31$ oct. 1978 , Bull. civ. III, $\mathrm{n}^{\circ} 329$. Pour des illustrations récentes, voir CA Paris, $3 \mathrm{e}$ ch., 9 octobre 2014, arrêt n ${ }^{\circ} 13$ 06037 qui refuse le jeu de l'exception d'inexécution ; CA Aix-en-Provence, 11e ch. B, 3 décembre 2015, arrêt n¹4-24013 qui l'admet.

${ }^{50}$ Comme par exemple, celle introduite par la loi ALUR dans l'art. 17-2 de la loi du 6 juillet 1989 et concernant l'action en réduction des loyers surévalués dans les zones comportant des loyers de référence. Ou bien encore celle également introduite par la loi ALUR à l'art. 3-1 de la loi de 1989 et relative à la diminution de loyer en cas de différence de plus d'1/20 entre la surface habitable mentionnée au bail et celle réellement constatée.

${ }^{51}$ A l'instar de l'ancienne rédaction du code, celle qui résulte de l'ordonnance de 2016 ne prévoit pas expressément de définition de la force majeure en matière extracontractuelle. Toutefois, celle-ci devrait logiquement intervenir lors de la réforme du droit de la responsabilité.
} 
En guise de conclusion, on ajoutera qu'il convient d'être attentif aux incidences de l'ordonnance sur le futur des baux en cours. Applicable depuis le $1^{\text {er }}$ octobre 2016, elle ne concerne certes que les baux conclus après cette date ${ }^{52}$. Mais, si une simple révision du loyer ne fera pas du bail un nouveau contrat soumis à la réforme, en revanche, tel ne sera pas le cas des reconductions. Parmi cellesci, les baux purement et simplement renouvelés par l'effet d'un statut appellent par prudence l'ajout ou la renégociation d'un certain nombre de clauses afin d'assurer leur adéquation avec les règles nouvelles issues de la réforme.

\footnotetext{
${ }^{52}$ L'art. 9 de l'ordonnance prend le soin de préciser que «les contrats conclus avant cette date demeurent soumis à la loi ancienne ».
} 